\title{
Poly(Lactic-co-glycolic) Acid and Phospholipids Hybrid Nanoparticles for Regeneration of Biological Tissue
}

\author{
Antonio Minó $^{1}\left(\mathbb{D}\right.$, Giuseppe Cinelli ${ }^{2} \mathbb{D}$, Gianluca Paventi ${ }^{3}\left(\mathbb{D}\right.$, Gianluca Testa $^{3}$, Fabiana Passaro ${ }^{4}(\mathbb{D}$, \\ Francesco Lopez $^{2} \mathbb{D}$ and Luigi Ambrosone ${ }^{3, * \mathbb{D}}$
}

1 Department of Biosciences and Territory (DiBT), University of Molise, Contrada Lappone, 86090 Pesche, Italy; a.mino@studenti.unimol.it

2 Dipartimento di Agricoltura Ambiente Alimenti (DiAAA), University of Molise, Via F. De Sanctis snc, 86100 Campobasso, Italy; giuseppe.cinelli@gmail.com (G.C.); lopez@unimol.it (F.L.)

3 Department of Medicine and Health Sciences "V. Tiberio", University of Molise, Via F. De Sanctis snc, 86100 Campobasso, Italy; paventi@unimol.it (G.P.); gianluca.testa@unimol.it (G.T.)

4 Department of Molecular Medicine and Medical Biotechnologies, University of Naples "Federico II", Via Pansini, 5, 80131 Napoli, Italy; fabiana.passaro@unina.it

* Correspondence: ambroson@unimol.it; Tel.: +39-0874-404715

check for updates

Citation: Minó, A.; Cinelli, G.; Paventi, G.; Testa, G.; Passaro, F.; Lopez, F.; Ambrosone, L. Poly(Lactic-co-glycolic) Acid and Phospholipids Hybrid Nanoparticles for Regeneration of Biological Tissue. ChemEngineering 2022, 6, 10.

https://doi.org/10.3390/ chemengineering6010010

Academic Editor: Alírio E. Rodrigues

Received: 27 November 2021

Accepted: 4 January 2022

Published: 27 January 2022

Publisher's Note: MDPI stays neutral with regard to jurisdictional claims in published maps and institutional affiliations.

Copyright: (C) 2022 by the authors. Licensee MDPI, Basel, Switzerland. This article is an open access article distributed under the terms and conditions of the Creative Commons Attribution (CC BY) license (https:// creativecommons.org/licenses/by/ $4.0 /)$.

\begin{abstract}
In tissue regeneration, biomaterials facilitate biological processes. However, a treatment with biomaterials will be successful only if supported by simple and inexpensive technologies which stimulate the regenerative processes. The present study focused on the possibility of creating formulations from which then to obtain suitable materials for the regeneration of heart tissue. The experimental procedure for precipitation of polymer- nanoparticles was modified ad hoc to obtain hybrid poly lactic-co-glycolic acid (PLGA)-phospholipid nanoparticles. The properties of the formulations produced by direct PLGA-phospholipid co-precipitation depend on the mass ratio $\mathcal{R}=$ polymer mass/phospholipid mass. The value of this parameter allows us to modulate the properties of the formulations. Formulations with $\mathcal{R}=1.5,2.3,4$, and 9 were prepared, and for each of them the particle-size distribution obtained by dynamic light scattering was studied. All samples showed that the hydrodynamic diameter decreases with increasing $\mathcal{R}$ value. This behavior is interpreted as polymer coil shrinkage due to contacts with the non-solvent. The spreadability and ease of obtaining thin sheets were evaluated for each formulation. The formulation with $\mathcal{R}=4$ resulted in a homogeneous and easily workable material in thin sheets.
\end{abstract}

Keywords: nanoparticles; PLGA; patch; phospholipids

\section{Introduction}

Poly lactic-co-glycolic acid (PLGA) is considered a suitable polymer for the fabrication of drug delivery devices and tissue engineering applications. PLGA is a biodegradable polymer approved by Food and Drug Administration [1]. In particular, PLGA has been extensively studied for the development of devices for the controlled delivery of small molecule drugs, proteins [2], and other macromolecules in research and commercial settings. In the last few decades, numerous protein-based bioactive polymers have been involved with delivery of molecules starting from the properties of the matrices structures formulated [3-7].

Currently, significant efforts are being made to integrate both diagnostic and therapeutic functions into a nanoscale system to achieve items associated with a wide range of scientific applications. Nanoparticles have great potential to achieve such purposes, especially when more than one type of nanostructure can be integrated into a nanoscale system [8]. Hybrid nanoparticles are composed of at least two different nanoparticles to overcome the limitations of single-component nanoparticles, as well as to recognize new properties not possible for single nanoparticles and to acquire additional functions [9-12]. 
To evaluate this type of systems, different mathematical approaches defining a specific model can be extremely useful and provide important information [13-17].

Experimentally, hybrid PLGA-phospholipid nanoparticles are generally fabricated either with the core-shell procedure [18] or by passive loading in liposomes [19]. Herein, we present results of hybrid particles obtained with a direct PLGA-phospholipid coprecipitation method. The rationale consists in finding easily reproducible, inexpensive and not very elaborate formulations for the development of materials useful in regenerative medicine. In this context, the use of biomaterials play the role of stimulating the regenerative processes. Indeed, regenerative medicine depends heavily on the development of tools that can help in repairing or replacing damaged or diseased human cells or tissues to restore normal function [20-23].

The discovery and development of erodible polymers that can be used with maximum efficiency and minimum risk to user requires the optimization of their transfer to the target during application [24]. The efficiency of the process crucially depends on the physical state of the formulation applied. Recently, a device consisting of liposomes trapped in cross-linked poly(lactic-co-glycolic) acid (PLGA) was suggested to overcome pulmonary hypertension problems [25]. The application of the device for the administration of the chemical cocktail for the regeneration of cardiac tissue involves a number of interfaces, where the interaction with the formulation plays a pivotal role.

Physicochemical parameters such as droplet size distribution, impaction and adhesion, sliding and retention, and wetting and spreading are of prime importance in ensuring maximum capture by the target surface as well as coverage of the target surface [26]. This research aims to identify a suitable formulation with specific mechanical properties for manufacturing a PLGA-based material capable of spreading on heart allowing tissue regeneration in order to deliver drugs.

\section{Materials and Methods}

\subsection{Chemicals}

Copolymer poly(D,L-lactide-co-glycolide) (Resomer RG $503 \mathrm{H}$ ) was purchased from Sigma Aldrich (St. Louis, MO, USA). The weight average molecular mass $\mathrm{M}_{w}$ was 24-38 kDa and the lactide/glycolide molar ratio 50:50. Dichloromethane $\mathrm{CH}_{2} \mathrm{Cl}_{2}(\mathrm{DCM})$ solvent was obtained from Sigma Aldrich. Phospholipids Epikuron 130P (EPK) composed of phosphatidylcholine (33\%), phosphatidylethanolamine (15\%), phosphatidylinositol $(16 \%)$, and phosphatidic acid (6\%) was generously donated by Cargill. UltraPure Water (UPW) (Milli Q system) was used in all measurements.

\subsection{Dynamic Light Scattering}

The average hydrodynamic diameter values of nanoparticles were determined at $25^{\circ} \mathrm{C}$ by means of dynamic light scattering (DLS) Malvern UK ZS90 commercial instrument operating with a $4 \mathrm{~mW}$ He-Ne laser (633 nm wavelength). The samples were dispersed into a suspension and loaded in a cuvette while the instrument was left free to optimize the instrumental parameters. The size distribution by intensity of scattered light was recovered, using the software implemented by the manufacturer at $25^{\circ} \mathrm{C}$. Each measurement was performed in triplicate.

\subsection{Preparation of Hybrid Nanoparticles}

Nanoprecipitation is a versatile method for making various types of polymer nanoparticles with well-controlled particle size [27]. PLGA masses of 60, 70, 80, and $90 \mathrm{mg}$ were dissolved in $5 \mathrm{~mL}$ of DCM then poured dropwise in the dispersing phase $\left(0.015 \mathrm{dm}^{3}\right.$ of UPW) by means of a syringe $(20 \mathrm{~mL})$ under moderate magnetic stirring, as shown in Figure 1. The suspension of nanoparticles then was sonicated for $1 \mathrm{~min}$ at $50 \% \mathrm{~W}$ and $50 \%$ pulser and left under stirring overnight. 


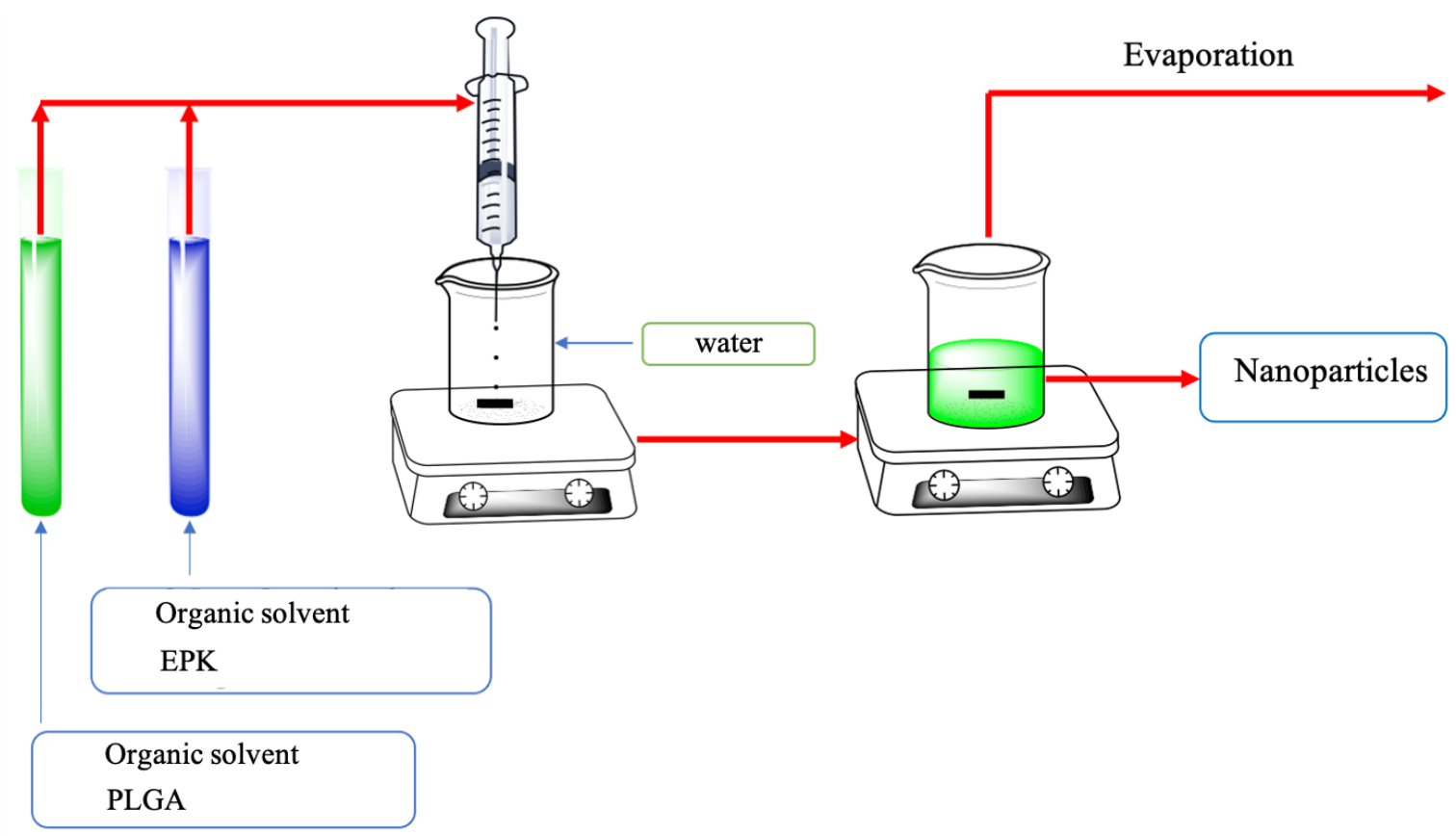

Figure 1. Process scheme for preparing mixed PLGA-EPK nanoparticles.

In order to prepare the hybrid nanoparticles, PLGA masses of 60, 70, 80, and $90 \mathrm{mg}$ were dissolved in $2.5 \mathrm{~mL}$ of DCM while 40, 30, 20, and $10 \mathrm{mg}$ of EPK were dissolved in other $2.5 \mathrm{~mL}$ of DCM. Both solutions were mixed in a syringe so as to obtain mixtures with a ratio $(\mathrm{m} / \mathrm{m})$ PLGA/EPK of 60:40, 70:30, 80:20, and 90:10.

The resulting mixture was poured dropwise $(1 \mathrm{~mL} / \mathrm{min})$ into $0.015 \mathrm{dm}^{3}$ of UPW which was stirred with a rotating magnet. Then, the suspension was sonicated for $1 \mathrm{~min}$ at $50 \% \mathrm{~W}$ and $50 \%$ pulser and left under stirring overnight.

\subsection{Preparation of Patches}

The freshly formed nanoparticles were separated from the precipitation environment and uniformly layered on slides and allowed to evaporate. When the solvent evaporation was completed, a patch material was deposited on the slide.

The adhesion strength of the patch was tested for each slide. By wetting the slide, the ability to detach while having shape remain intact was evaluated. Furthermore, the workability of the material, i.e., the detachment from the slide and its accommodation on another surface, was evaluated.

\section{Results and Discussion}

The DLS size distributions of all control nanoparticles are shown in Figure 2a. The spectra represent log-normal distributions with maximum values centered in the 150-300 nm size range. To check whether free energy, $G^{\text {form }}$, of the system, is sufficient to cause a size distribution, a rough estimate can be made from considering the balance between the entropy of dispersion and the interfacial energy [28], i.e.,

$$
\Delta G^{\text {form }}=\gamma \Delta A-T \Delta S^{\mathrm{conf}}
$$

where $\gamma$ is the interfacial tension between the two phases, $\Delta A$ is the increase in interfacial area, $T$ is the absolute temperature and $\Delta S^{\text {conf }}$ is the configurational entropy in the resulting dispersion. The first term, $\gamma \Delta A$, is the work required to expand the interface and it is positive. The second term, $T \Delta S^{\text {conf }}$, is the configurational entropy resulting from the production of a number of particles. The formation of new nanoparticles implies an increase in $\Delta A$ so it must be $|\gamma \Delta A|<T\left|\Delta S^{\text {conf }}\right|$ for the nanoparticles to form spontaneously. This is achieved because the long and flexible molecules of PLGA in the presence of a poor 
solvent, such as water, curl up assuming the configuration of random coil [29]. In this process, a large number of solvent molecules are released and the entropy of the system increases. The random coil configuration adopted by the PLGA molecules depends both on molecular mass of the polymer and its concentration [30]. On the other hand, each PLGA rearrangement corresponds to a hydrodynamic particle diameter, $d_{z}$, of the nanoparticles produced. Figure $2 \mathrm{~b}$ shows that the large reduction in $d_{z}$ is simply a consequence of the increase in PLGA concentration. Moreover, for $C_{\mathrm{PLGA}} \approx 6 \mathrm{gdm}^{-3}, d_{z}$ undergoes a sort of "collapse". This is due to the fact that at relatively low PLGA concentration, the monomers avoid each other and particle is kept large to maximize its conformational entropy. At higher PLGA concentration, the proximity between the monomers leads to hydrophobic attraction which promotes particle collapse. Note that the sizes of PLGA obtained in this research are compatible with those obtained in acetonitrile $(114 \pm 24 \mathrm{~nm})$, vortexed and filtered [18].
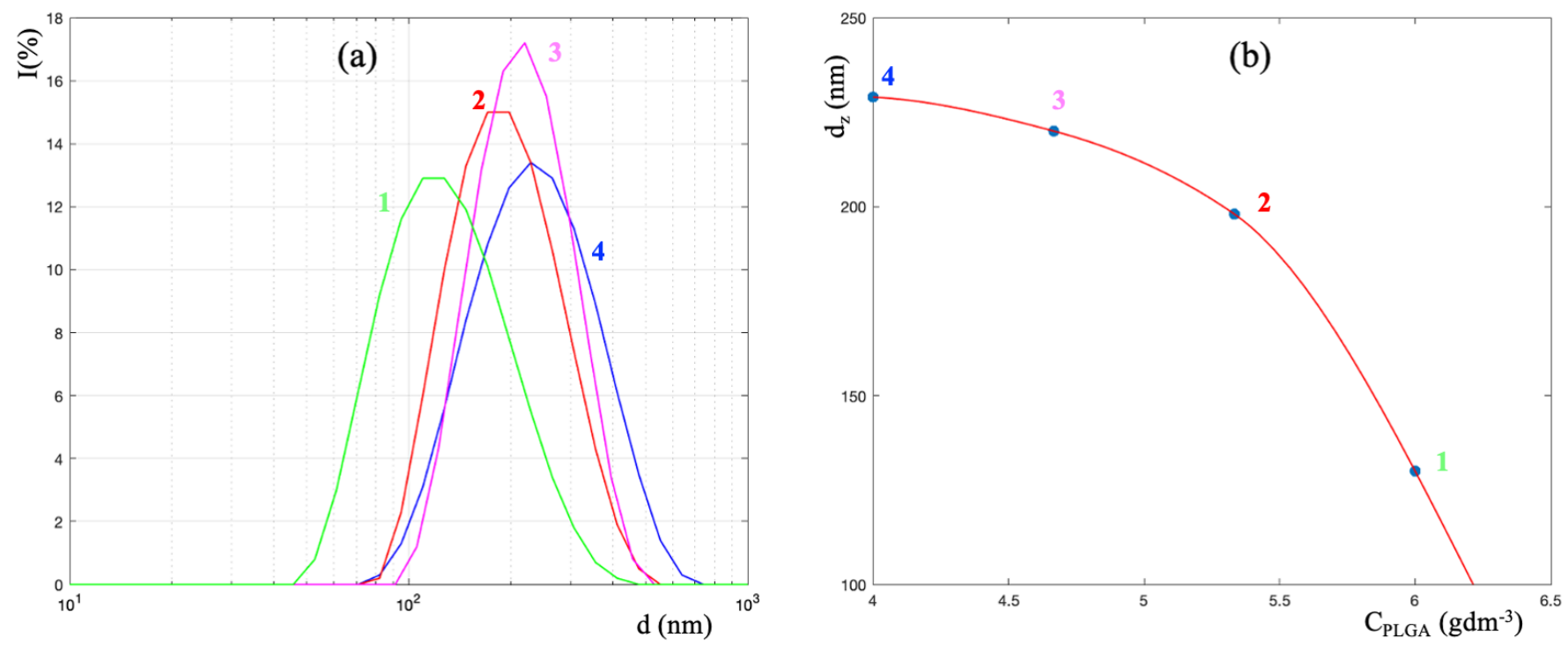

Figure 2. (a) Particle size distribution of PLGA nanoparticles obtained by nanoprecipitation. (b) Z-average of PLGA nanoparticles as a function of the polymer concentration.

When the formation of nanoparticles takes place in a medium containing EPK, the physicochemical process is somewhat more complex. In fact, the process of break-up of liquid sheets into nanoparticles seems to be the result of surface forces tending to divide the system into spherical particles. In order to study the formation process of hybrid nanoparticles, the parameter $\mathcal{R}$ was defined as follows:

$$
\mathcal{R}=\frac{\operatorname{mass}(\mathrm{PLGA})}{\operatorname{mass}(\mathrm{EPK})}=\frac{C_{\mathrm{PLGA}}}{C_{\mathrm{EPK}}}
$$

$\mathcal{R}$ was varied experimentally from 1.5 to 9 and each formulation was analyzed by DLS. For the different $\mathcal{R}$ ratios, it ensured that in the resulting mixtures there was the same amount of polymer, as in PLGA nanoparticles. Furthermore, the amount of phospholipid was that generally used to prepare phosphatidylcholine liposomes [31]. Figure 3a shows the measured particle size distributions. Although thermodynamics determines the most stable configuration of a system (see Equation (1)), in real systems, due to the different process rates, metastable situations are created. In the formation of hybrid nanoparticles, if the formation rate of the PLGA-water interface is much faster than the formation rate of the EPK-water interface, the EPK molecules will accumulate on the PLGA spheres. On the other hand, if it is the formation rate of the EPK-water interface that is faster, then the lipid will aggregate and incorporate the PLGA particles. 
It can be clearly seen in Figure $3 a$ that by increasing the parameter $\mathcal{R}$, the particle size distributions exhibit complex shapes, for $\mathcal{R}=1.5$ the size distribution of hybrid nanoparticles is only slightly different from reference, for $\mathcal{R}=2.3$ the size distribution tends to widen whilst, for $\mathcal{R}=4$ it becomes bimodal. Finally, for $\mathcal{R}=9$, it is again unimodal with smaller average dimensions.
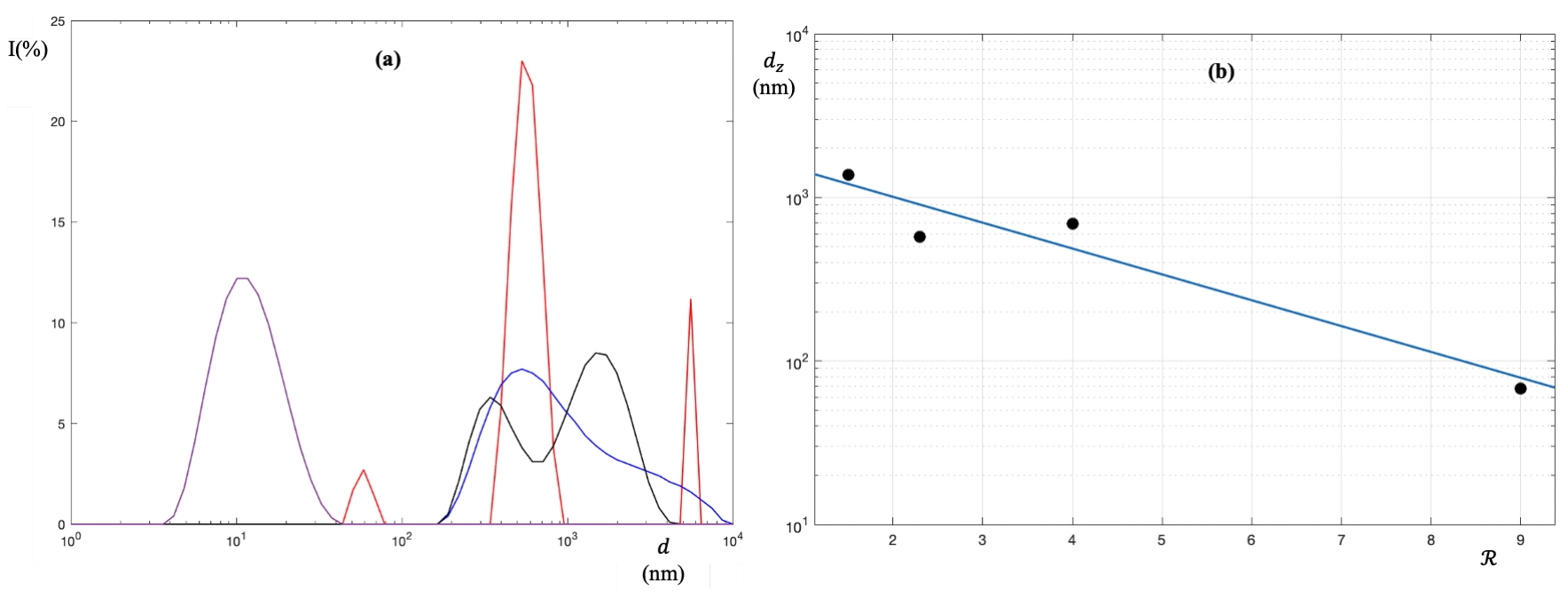

Figure 3. (a) Particle size distributions of mixed PLGA-EPK dispersions as measured by DLS. Purple curve $\mathcal{R}=1.5$, blue curve $\mathcal{R}=2.3$, black curve $\mathcal{R}=4$, and red curve $\mathcal{R}=9$. (b) Semi-log plot of $\mathrm{Z}$-average as a function of the ratio $\mathcal{R}$, linearity indicates that the $d_{z}$ decreases exponentially.

This complex response of the system can be better studied by considering $d_{z}$ as a function of $\mathcal{R}$. Figure $3 \mathrm{~b}$ reveals that a semi-log plot, with a linear $X$ axis, of $d_{z}$ vs. $\mathcal{R}$ exhibits a linear trend. Therefore, the regression procedure provides the following relationship:

$$
d_{z}=1600 e^{-0.3 \mathcal{R}} \quad(\mathrm{nm})
$$

The large decrease in $d_{z}$ can be explained by the shrinkage of the PLGA coil. Indeed, an increase in $\mathcal{R}$ leads to a larger number of polymer-water molecule contacts, which cause further shrinkage of the polymer molecules. Accordingly, the hydrodynamic diameter is reduced.

Note that hybrid nanoparticles obtained by precipitation in water of Fl-ceramideloaded PLGA and combined with liposomes exhibit $d_{z}=195 \pm 15 \mathrm{~nm}$ [19]. Although the procedure used is very complex and the solvent is different, the size of these nanoparticles is in the range shown in Figure 3b. This suggests that a simple direct PLGA-phospholipid co-precipitation method is capable of delivering nanoparticles with dimensions comparable to other methods. Furthermore, by varying the $\mathcal{R}$ ratio, it is possible to modulate the diameter of the nanoparticles.

The goal of the formulations is to provide a simple procedure for producing a durable material adhering to tissues and easy to be handled. For this reason, each formulation was separated from its precipitating medium and spread on a slide. The shape of the residual material left behind when the liquid phase evaporates, depends largely on the $\mathcal{R}$ ratio used to prepare the formulations. An important factor in the deposited material is the tenacity. Indeed, strong adhesion between material and the support-slide creates difficulties in removal and workability. During evaporation, liquid crystals of very high viscosity may be produced. Such highly viscous structures can trap low molecular mass compounds and serve as reservoirs for chemicals. The material derived from the formulations with $\mathcal{R}=1.5$ or $\mathcal{R}=2.3$ is not spreadable on the slide and crumbles in heterogeneous pieces which are difficult to use (inset Figure 4a). An aliquot of the material was removed from the slide and resuspended in water giving the size spectrum displayed in Figure 4a. As one can 
see, the distribution exhibits three distinct peaks indicating difficult rehydration and poor exfoliation of the material.
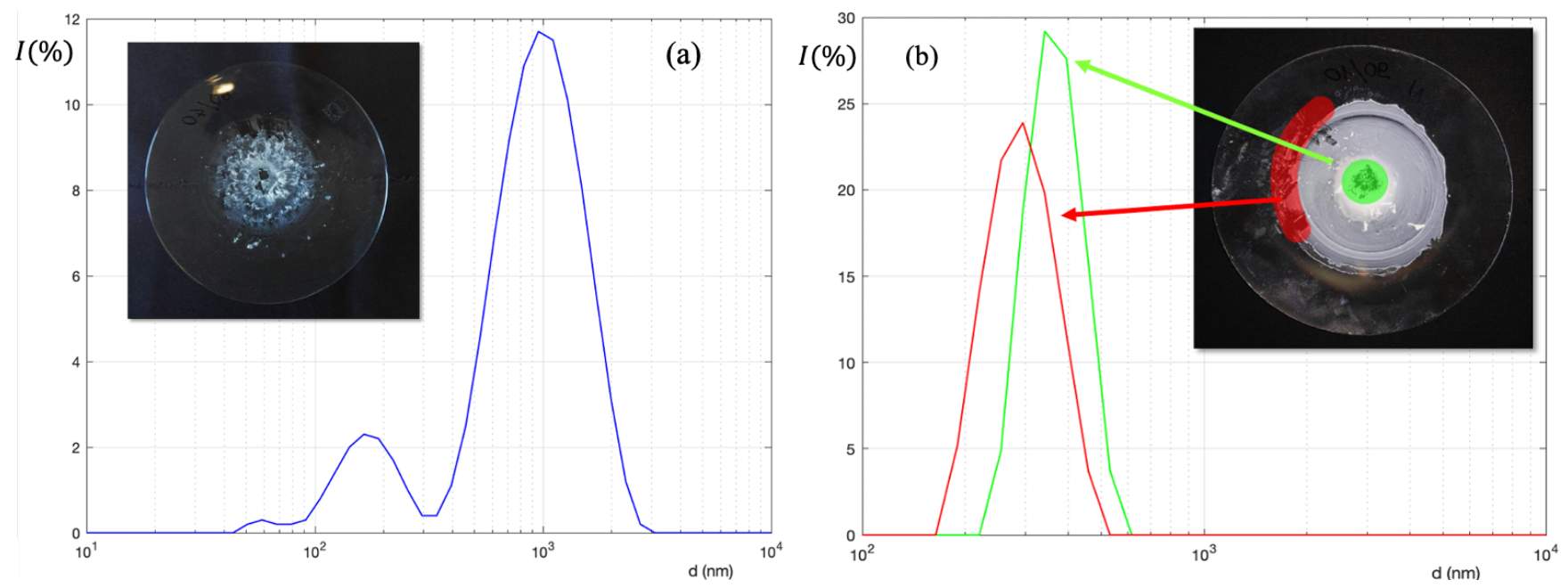

Figure 4. (a) Material obtained by rehydration of the formulation $\mathcal{R}=1.5$. The droplet size distribution of the rehydrated sample shows a large polydispersity indicating an inhomogeneity of the dispersion. The inset shows that the material is not spreadable on a glass slide. (b) Material obtained by rehydration of the formulation $\mathcal{R}=9$. The droplet size distribution of the rehydrated sample shows a low polydispersity, indicating a good homogeneity of the dispersion. The inset shows that the material is waxy and spreadable on a glass slide, material samples taken from two different points on the slide, after rehydration, exhibit similar droplet size distribution.

Evaporation of liquid phase from formulation with $\mathcal{R}=9$, produces a waxy material which is easily spreadable on a slide. The material is visually homogeneous and the degree of homogeneity was estimated as follows. Two samples were taken from the dry sample placed on the slide. The first on the outer edge, the second in the center of the slide, as shown in the inset of Figure $4 \mathrm{~b}$. The collected samples were rehydrated individually and analyzed by DLS. As it can be seen in Figure $4 \mathrm{~b}$ both particle size distributions are sufficiently narrow and very close, indicating a good homogeneity of the sample. However, due to its high adhesiveness, it hardly comes off the slide. This property makes it unsuitable for the developing patches to be applied to biological tissues. Finally, the material obtained from the formulation with $\mathcal{R}=4$, although it does not have a waxy consistency, can be evenly and easily distributed on a slide. Moreover, the material is homogeneous and can be detached by simply wetting the slide, as shown in Figure 5a. The material detached from the slide retains the shape of the film and bends easily without breaking. These properties are good requirements for the development of a material to be used as drug delivery patch for tissue regeneration. In this sense, a $2.5 \times 6 \mathrm{~cm}^{2}$ sample patch was fabricated to perform preliminary tests of accomodability and adaptability to cardiac tissue, Figure $5 \mathrm{~b}$. Although these first results seem to indicate a good response of the material, further experiments are needed for a full discussion of the application of the material on animal heart. 

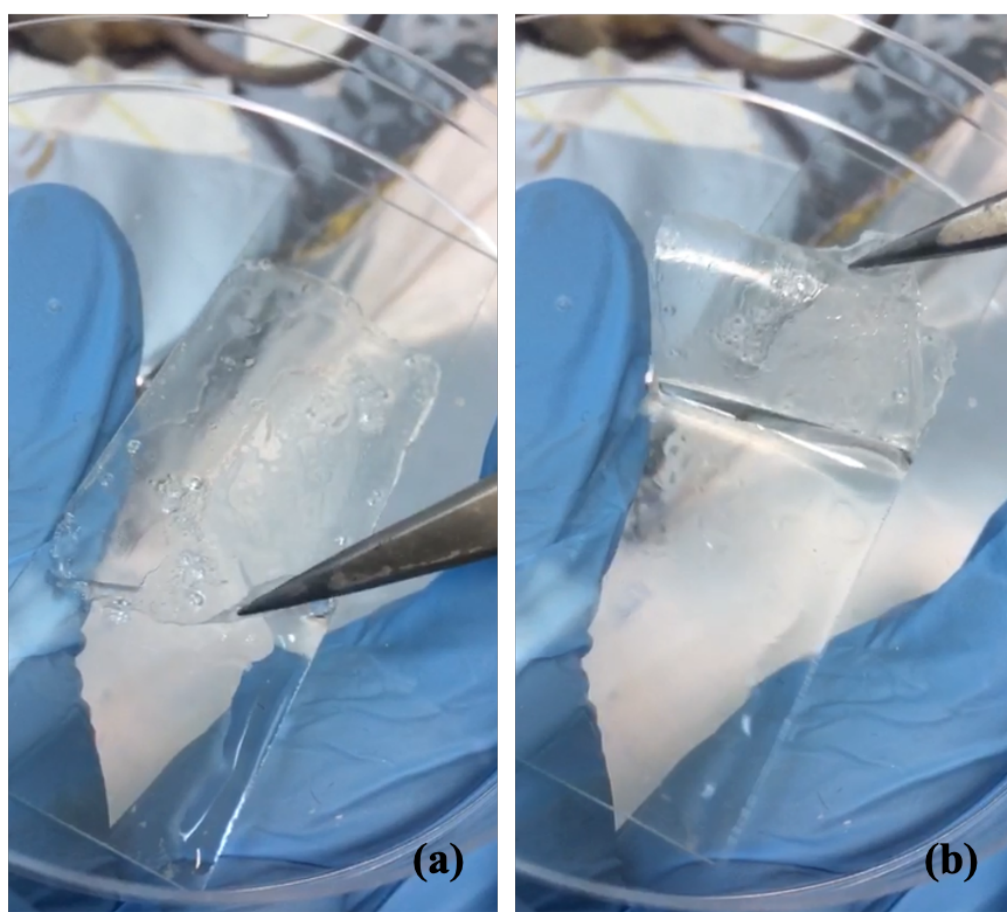

Figure 5. Patch manufactured from the formulation $\mathcal{R}=4$. (a) The material is easily detachable from the slide, by simple hydration. (b) The material is very flexible and easily adaptable to biological tissues.

\section{Conclusions}

The process, generally used for the nanoprecipitation of polymers, was modified ad hoc to obtain hybrid nanoparticles of PLGA and EPK. By varying the ratio $\mathcal{R}$, hybrid nanoparticles with different chemical-physical properties are obtained. The DLS measurements performed for each formulation show that the size spectrum takes a very complex shape depending on several factors. However, the larger $\mathcal{R}$, the smaller the mean hydrodynamic diameter of the nanoparticles. The reduction in diameter is due to the contacts between the polymer and use of a poor solvent such as water. A new solution containing hybrid particles eventually useful for regeneration procedures was obtained from each formulation by evaporating the liquid phase. The performance of this material depends on the $\mathcal{R}$ value of the starting formulation. In particular, the material obtained by the formulation with $\mathcal{R}=4$ presents chemical characteristics making it suitable for surgical use.

Although further measurements are needed, it appears that the material obtained in this research constitutes the starting point for obtaining material to be used in regenerative medicine for the regeneration of heart tissue.

Author Contributions: Conceptualization, methodology, L.A., F.L. and G.T.; software, G.C., G.P. and L.A.; formal analysis, L.A. and A.M.; investigation, A.M., G.C. and F.P.; data curation, G.P., G.C. and L.A.; writing-original draft preparation, L.A., G.T. and A.M.; project administration, L.A. and F.L. All authors have read and agreed to the published version of the manuscript.

Funding: This research received no external funding.

Acknowledgments: The author thank Giuseppe D'Amato for the IT support during the drafting of the paper.

Conflicts of Interest: The authors declare no conflicts of interest.

\section{References}

1. Makadia, H.K.; Siegel, S.J. Poly lactic-co-glycolic acid (PLGA) as biodegradable controlled drug delivery carrier. Polymers 2011, 3, 1377-1397. [CrossRef]

2. Hawkins, M.J.; Soon-Shiong, P.; Desai, N. Protein nanoparticles as drug carriers in clinical medicine. Adv. Drug Deliv. Rev. 2008, 60, 876-885. [CrossRef] [PubMed] 
3. Azimi, B.; Nourpanah, P.; Rabiee, M.; Arbab, S. Producing gelatin nanoparticles as delivery system for bovine serum albumin. Iran. Biomed. J. 2014, 18, 34.

4. Naderi-Meshkin, H.; Andreas, K.; Matin, M.M.; Sittinger, M.; Bidkhori, H.R.; Ahmadiankia, N.; Bahrami, A.R.; Ringe, J. Chitosanbased injectable hydrogel as a promising in situ forming scaffold for cartilage tissue engineering. Cell Biol. Int. 2014, $48,72-84$. [CrossRef]

5. Lopez, F.; Venditti, F.; Ambrosone, L.; Colafemmina, G.; Ceglie, A.; Palazzo, G. Gelatin microemulsion-based gels with the cationic surfactant cetyltrimethylammonium bromide: A self-diffusion and conductivity study. Langmuir 2004, 20, 9449-9452. [CrossRef]

6. Ghidoni, I.; Chlapanidas, T.; Bucco, M.; Crovato, F.; Marazzi, M.; Vigo, D.; Torre, M.L.; Faustini, M. Alginate cell encapsulation: New advances in reproduction and cartilage regenerative medicine. Cytotechnology 2008, 58, 49-56. [CrossRef] [PubMed]

7. Lopez, F.; Venditti, F.; Cinelli, G.; Ceglie, A. The novel hexadecyltrimethylammonium bromide (CTAB) based organogel as reactor for ester synthesis by entrapped Candida rugosa lipase. Process Biochem. 2006, 41, 114-119. [CrossRef]

8. Sailor, M.J.; Park, J.-H. Hybrid nanoparticles for detection and treatment of cancer. Adv. Mater. 2012, 24, 3779-3802. [CrossRef]

9. Ma, D. Hybrid nanoparticles: An introduction. Noble Metal-Metal Oxide Hybrid Nanoparticles; Elsevier: Amsterdam, The Netherlands, 2019; pp. 3-6.

10. Teekamp, N.; Duque, L.F.; Frijlink, H.W.; Hinrichs, W.L.J.; Olinga, P. Production methods and stabilization strategies for polymerbased nanoparticles and microparticles for parenteral delivery of peptides and protein. Expert Opin. Drug Deliv. 2015, 12, 1311-1331. [CrossRef]

11. Lee, P.-C.; Zan, B.-S.; Chen, L.-T.; Chung, T.-W. Multifunctional PLGA-based nanoparticles as a controlled release drug delivery system for antioxidant and anticoagulant therapy. Int. J. Nanomed. 2019, 14, 1533. [CrossRef]

12. Soares, D.C.F.; Domingues, S.C.; Viana, D.B.; Tebaldi, M.L. Polymer-hybrid nanoparticles: Current advances in biomedical applications. Biomed. Pharmacother. 2020, 131, 110695. [CrossRef] [PubMed]

13. Cinelli, G.; Bufalo, G.; Lopez, F.; Ambrosone, L. Cooperativity between Dimerization and Binding Equilibria in the Ternary System Laponite-Indocyanine Green-Water. ChemEngineering 2021, 5, 6. [CrossRef]

14. Bufalo, G.; Florio, C.; Cinelli, G.; Lopez, F.; Cuomo, F.; Ambrosone, L. Principles of minimal wrecking and maximum separation of solid waste to innovate tanning industries and reduce their environmental impact: The case of paperboard manufacture. $J$. Clean. Prod. 2018, 174, 324-332. [CrossRef]

15. Ambrosone, L.; Cinelli, G.; Mosca, M.; Ceglie, A. Susceptibility of water-emulsified extra virgin olive oils to oxidation. J. Am. Oil Chem. Soc. 2006, 83, 165-170. [CrossRef]

16. Di Biasio, A.; Ambrosone, L.; Cametti, C. Numerical simulation of dielectric spectra of aqueous suspensions of non-spheroidal differently shaped biological cells. J. Phys. D Appl. Phys. 2008, 42, 401-410. [CrossRef]

17. Molino, B.; Bufalo, G.; De Vincenzo, A.; Ambrosone, L. Semiempirical model for assessing dewatering process by flocculation of dredged sludge in an artificial reservoir. Appl. Sci. 2020, 10, 3051. [CrossRef]

18. Chan, J.M.; Zhang, L.; Yuet, K.P.; Liao, G.; Rhee, J.W.; Langer, R.; Farokhzad, O.C. PLGA-lecithin-PEG core-shell nanoparticles for controlled drug delivery. Biomaterials 2009, 30, 1627-1634. [CrossRef]

19. Zou, P.; Stern, S.T.; Sun, D. PLGA/liposome hybrid nanoparticles for short-chain ceramide delivery. Pharm. Res. 2014, 31, 684-693. [CrossRef]

20. Ciccocioppo, R.; Cantore, A.; Chaimov, D. Regenerative medicine: The red planet for clinicians. Intern. Emerg. Med. 2019, 14, 911-921. [CrossRef]

21. Passaro, F.; Testa, G.; Ambrosone, L.; Costagliola, C.; Tocchetti, C.B.; Di Nezza, F.; Russo, M.; Pirozzi, F.; Abete, P.; Russo, T. Nanotechnology-based cardiac targeting and direct cardiac reprogramming: The Betrothed. Stem Cells Int. 2017, $2017,4940397$. [CrossRef]

22. Passaro, F.; Tocchetti, C.G.; Spinetti, G.; Paudice, F.; Amborsone, L.; Costagliola, C.; Cacciatore, F.; Abete, P.; Testa, G. Targeting fibrosis in the failing heart with nanoparticles. In Advanced Drug Delivery Reviews; Elsevier: Amsterdam, The Netherlands, 2021.

23. Singh, R.; Lillard, J.W., Jr. Nanoparticle-based targeted drug delivery. Exp. Mol. Pathol. 2009, 86, 215-223. [CrossRef]

24. Guan, J.; Chen, W.; Yang, M.; Wu, E.; Qian, J.; Zhan, C. Regulation of in vivo delivery of nanomedicines by herbal medicines. In Advanced Drug Delivery Reviews; Elsevier: Amsterdam, The Netherlands, 2021.

25. Testa, G; Ambrosone, L; Costagliola, C. Biodegradable Polymer Medical Device. European Patent n. PCT/IB2015/059278, 2 December 2015.

26. Baldelli, A.; Ou, J.; Li, W.; Amirfazli, A. Spray-On Nanocomposite Coatings: Wettability and Conductivity. Langmuir 2020, 36, 11393-11410. [CrossRef] [PubMed]

27. Beck-Broichsitter, M.; Rytting, E.; Lebhardt, T.; Wang, X.; Kissel, T. Preparation of nanoparticles by solvent displacement for drug delivery: A shift in the "ouzo region" upon drug loading. Eur. J. Pharm. Sci. 2010, 41, 244-253. [CrossRef]

28. Costagliola, C.; Semeraro, F.; Zeppa, L.; Bufalo, G.; Cardone, M.; Romano, M.; Ambrosone, L. Some physicochemical remarks on spontaneous emulsification of vitreal tamponades. BioMed Res. Int. 2014, 2014, 243056. [CrossRef] [PubMed]

29. Fujita, H. Polymer Solutions; Elsevier: Amsterdam, The Netherlands, 2012.

30. Uehara, E.; Deguchi, T. Statistical and hydrodynamic properties of topological polymers for various graphs showing enhanced short-range correlation. J. Chem. Phys. 2016, 145, 164905. [CrossRef] [PubMed]

31. Mosca, M.; Ceglie, A.; Ambrosone, L. Effect of membrane composition on lipid oxidation in liposomes. Chem. Phys. Lipids 2011, 164, 158-165. [CrossRef] [PubMed] 